\title{
Distress Screening and Management in Early Breast Cancer Patients: Distress after Breast Cancer Diagnosis and Associated Factors
}

\author{
Hyunnam Baek, Eunyoung Kang ${ }^{1}$, Angela Soeun Lee ${ }^{1}$, Euijun Hwang ${ }^{1}$, Sumin Chae ${ }^{1}$, Eun-Kyu Kim¹, \\ Sung-Won Kim ${ }^{2}$ \\ Department of Surgery, Sahmyook Seoul Medical Center, Seoul; 'Department of Surgery, Seoul National University Bundang Hospital, Seoul National \\ University College of Medicine, Seongnam; ${ }^{2}$ Department of Surgery, Daerim St. Mary's Hospital, Seoul, Korea
}

Purpose: The aims of this study were to evaluate the magnitude of distress after breast cancer diagnosis and to investigate factors associated with distress, as well as to determine the effectiveness of psychological intervention. Methods: This study was performed retrospectively, reviewing 264 patients who underwent breast cancer surgery at Seoul National University Bundang Hospital between November 2011 and May 2014. Distress was measured using the distress thermometer (DT) and Center for Epidemiological Studies-Depression scale (CES-D) questionnaires before, as well as at 3 and 6 months postsurgery. Psychological intervention was recommended to high risk patients (DT score $\geq 5$ or CES-D score $\geq 16$ ). Results: In total, 149 patients (56.4\%) were classified as high risk in the initial assessment. In the following assessments, the proportion of those in the high risk group was $38.5 \%$ and $25.0 \%$ at 3 and 6 months postsurgery, respectively. Mastectomy was significantly associated with high levels of distress compared to breast-conserving surgery in the univariate $(p=0.048)$ and multivariate analyses $(p=0.014)$. However, there was no significant relationship between any of the various socioeconomic factors and distress. Distress level was reduced over time in both scales. Of the 149 high risk patients, only 21 received the psychological intervention. Using linear mixed models, the psychological intervention resulted in marginally significant reductions in DT $(p=0.051)$ and CES-D $(p=0.077)$ scores. Conclusion: More than half of patients experienced distress upon initial diagnosis, and the determined surgery type was an important factor associated with high distress level. It is important to identify high risk patients and to manage distress during the initial phase.

Key Words: Breast neoplasms, Distress, Psychological intervention

\section{INTRODUCTION}

Breast cancer is one of the most common cancers affecting women worldwide. In Korea, approximately 16,000 people were diagnosed with breast cancer in 2010. The 5-year survival rate for breast cancer patients has also increased to $91.0 \%$ due to early detection and treatment advancements [1]. Hence, the goal of breast cancer treatments is not only to maximize survival, but also to improve the quality of life for cancer survivors.

In general, the majority of cancer patients have experienced stress after cancer diagnosis, and about one-third of cancer patients have ex-

\section{Correspondence: Eunyoung Kang}

Department of Surgery, Seoul National University Bundang Hospital, Seoul National University College of Medicine, 82 Gumi-ro 173beon-gil, Bundang-gu, Seongnam 13620, Korea

Tel: +82-31-787-7102, Fax: +82-31-787-4078, E-mail: key5002@gmail.com

The present research has been supported by the Korea Breast Cancer Foundation.

Received: Nov 8, 2016 Revised: Dec 28, 2016 Accepted: Feb 12, 2017 perienced clinically significant stress or mental dysfunction $[2,3]$. According to a study that assessed new mental disorders and stress among patients with a breast cancer diagnosis, approximately $41 \%$ of women had experienced clinically significant stress, $11 \%$ suffered from major depression, and 10\% suffered from posttraumatic stress disorder [4]. Therefore, distress screening and management for cancer patients is becoming increasingly recognized as important and, thus, recommended [5]. The National Comprehensive Cancer Network (NCCN) already suggested guidelines for managing distress in 1999, by means of evaluating the degree of mental illness in all patients during the follow-up period and providing them to an initial screening [6]. In addition to screening, many psychosocial intervention programs have been developed and tested. The effectiveness of these psychosocial interventions in reducing emotional distress has been demonstrated and proven in many studies [7-9].

The aims of this study were to evaluate the level of distress after breast cancer diagnosis, to investigate the factors associated with distress, to evaluate changes in distress levels according to time, and to 
evaluate the effect of a psychological intervention.

\section{METHODS}

\section{Study design}

Between November 2011 and July 2014, a total of 412 patients were screened to undergo breast cancer surgery at Seoul National University Bundang Hospital. Patients with preoperative chemotherapy or stage III/IV breast cancer $(n=73)$, with a prior breast cancer history $(n=62)$, and with incomplete written questionnaires $(n=13)$, were excluded. We analyzed the data on 264 patients with stage $0 / \mathrm{I} / \mathrm{II}$ breast cancer. Clinical data were collected through a medical record review, which included clinico-pathological features, personal medical history, family history of breast/ovary/other cancer, socioeconomic status, and social history. All patients filled out two types of questionnaires, the distress thermometer (DT) and the Center for Epidemiological
Studies-Depression scale (CES-D), at three different points in time to evaluate the risk of distress. The first measurement was taken before surgery (T0), the second measurement was taken during an outpatient visit 3 months after surgery (T3), and the third measurement was taken after 6 months (T6) (Figure 1). This study was reviewed and approved by the Institutional Review Board of Seoul National University Bundang Hospital (IRB number: B-1407/258-113).

\section{Questionnaires}

The DT, recognized by the NCCN Psychosocial Distress Practice Guidelines Panel [10], is a brief self-report screening tool that measures the sources of distress over the past week. The DT is a visual analogue scale with scores ranging from 0 (no distress) to 10 (extreme distress). We considered a DT score of 5 or greater as an indication of clinically significant distress. The CES-D is a 20 -item self-report scale, measuring the incidence and degree of depressive symptoms over the

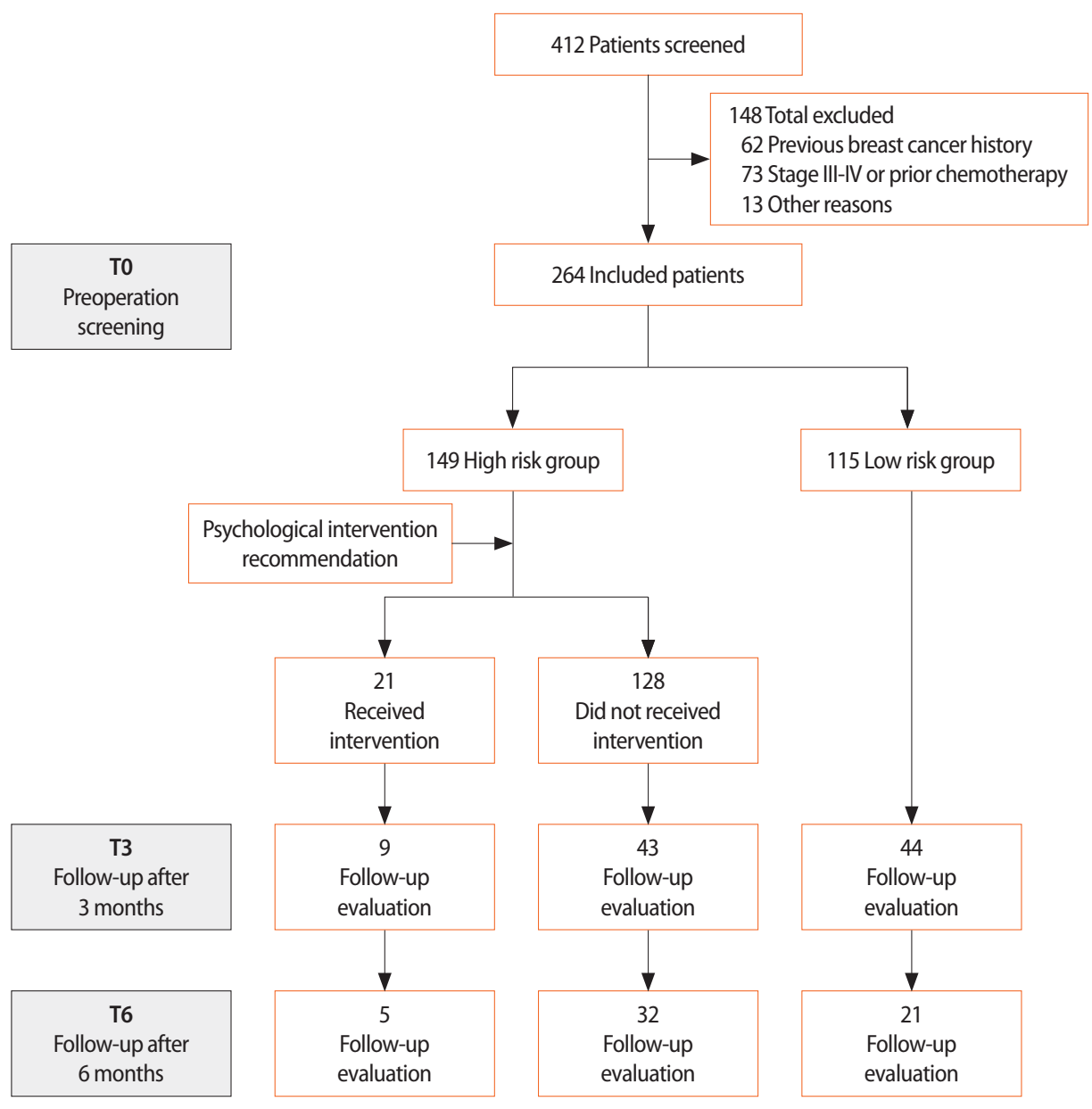

Figure 1. The study flow scheme. 
past week. The rating scale ranges from 0 (seldom or never) to 3 (almost or always), and scores can range from 0 to 60 [11]. The CES-D has been established as a valid and reliable measure of depressive symptoms in breast cancer patients $[12,13]$. We considered a CES-D score of $>16$ as an indication of clinically significant distress.

\section{Additional procedures}

All patients who were scheduled for breast cancer surgery completed the DT and CES-D questionnaires during their preoperative outpatient visits. We classified patients as high risk if one of the two scales exceeded the aforementioned criteria. Patients were provided psychological support by professionally trained nurses during preoperative counseling. We recommended that high risk patients participate in the psychological intervention. For patients who agreed to use the stress clinic within the breast cancer center, the intervention consisted mainly of supportive counseling conducted by psychiatrists. Cognitive behavioral therapy and psychopharmacologic intervention were implemented according to the patient's condition. If necessary, the intervention was continuously performed.

\section{Statistical analysis}

Data were analyzed by using SPSS software version 22.0 (IBM Corp., Armonk, USA). Binary logistic regression was used for comparison of categorical variables for univariate and multivariate analysis. Linear mixed models were used to analyze the change over time and the effect of intervention. The average the DT and the CES-D scores of the low risk and high risk groups were compared using the Mann-Whitney test. A $p$-value of $<0.05$ was considered to be statistically significant.

\section{RESULTS}

We analyzed 264 early breast cancer patients with a mean age of 51.7 years (range, 26-83 years). One hundred forty-nine patients (56.4\%) were classified as high risk in the initial screening assessment. It was recommended that all patients in the high risk group receive psychological intervention, but only 21 (14.1\%) received treatment. After 3 months, 96 patients underwent a follow-up assessment, and 37 (38.5\%) were evaluated as high risk. After 6 months, 60 patients were evaluated, and 15 (25.0\%) were considered high risk.
The low risk and high risk groups were compared on various factors, including sociodemographic and medical factors at T0, which were analyzed using univariate and multivariate analyses (Table 1). Mastectomy was significantly associated with high levels of distress compared to breast-conserving surgery (BCS) in the univariate analysis $(p=0.049)$. In the multivariate analysis, mastectomy was also identified as a risk factor $(p=0.047)$ for high distress. However, there was no significant relationship between the other factors and distress.

Additional analyses were conducted comparing mastectomy patients who underwent reconstructive surgery to those who did not, as well as to those who only had BCS. At T0, 178 patients (67.4\%) underwent BCS, 57 (21.6\%) underwent mastectomy only, and 29 (11.0\%) underwent mastectomy with reconstruction. All three groups showed overall decreasing levels of distress as measured via the distress scales, and those in the reconstruction group showed the most reduction in distress at T3 (Figure 2).

Distress levels were highest immediately before the surgery, and as the treatment proceeded, distress gradually decreased (Figure 3). The DT average score was 4.23 (standard deviation [SD], 2.71) at T0 $(\mathrm{n}=264), 3.19(\mathrm{SD}, 2.56)$ at T3 $(\mathrm{n}=96)$, and $2.53(\mathrm{SD}, 2.13)$ at T6 $(\mathrm{n}=58)$. The CES-D average score was $17.55(\mathrm{SD}, 12.27)$ at T0, 12.85 (SD, 12.04) at T3, and 6.40 (SD, 8.24) at T6.

In this study, 21 of the 149 (14.1\%) initially high risk patients received psychological intervention by a psychiatrist in the stress clinic. Twenty-one received psychiatric support and 12 were prescribed antidepressants and antianxiety medications. Nine visited the stress clinic only once and 12 visited twice more. We analyzed the distress scores between the intervention group and nonintervention group. At T0 $(\mathrm{n}=149)$, the average score of the intervention group was 7.30 (SD, 1.76) on DT and 31.67 (SD, 12.73) on CES-D. Conversely, the average score of the nonintervention group was 5.78 (SD, 2.16) on DT and 24.00 (SD, 9.55) on CES-D. The average score on the screening test between the two groups showed a significant difference (DT, $p=0.002$; $\mathrm{CES}-\mathrm{D}, p=0.01)$. As time passed, the scores of both groups decreased (DT, $p=0.003$; CES-D, $p=0.001$ ), and the effect of the intervention tended to be significant (DT, $p=0.051$; CES-D, $p=0.077$ ) (Table 2).

\section{DISCUSSION}

This study identified that the prevalence of preoperative distress is 
Table 1. Patients' characteristics: comparison between high and low distress groups

\begin{tabular}{|c|c|c|c|c|c|c|c|}
\hline \multirow{2}{*}{ Factor } & \multirow{2}{*}{$\begin{array}{c}\text { All patients } \\
(n=264) \\
\text { No. }(\%)\end{array}$} & \multirow{2}{*}{$\begin{array}{l}\text { High distress group } \\
\qquad(n=149) \\
\text { No. }(\%)\end{array}$} & \multirow{2}{*}{$\begin{array}{l}\text { Low distress group } \\
\qquad \begin{array}{c}(n=115) \\
\text { №. }(\%)\end{array}\end{array}$} & \multicolumn{2}{|c|}{ Univariate analysis } & \multicolumn{2}{|c|}{ Multivariate analysis } \\
\hline & & & & OR $(95 \% \mathrm{Cl})$ & $p$-value & OR $(95 \% \mathrm{Cl})$ & $p$-value \\
\hline \multicolumn{8}{|l|}{ Age (yr) } \\
\hline$<41$ & $21(8.0)$ & $12(8.1)$ & $9(7.8)$ & 1.00 & 0.497 & 1.00 & 0.434 \\
\hline $41-50$ & $103(39.0)$ & $61(40.9)$ & $42(36.5)$ & $1.09(0.42-2.82)$ & 0.860 & $2.38(0.70-8.05)$ & 0.165 \\
\hline $51-60$ & $85(32.2)$ & $50(33.6)$ & $35(30.4)$ & $1.07(0.41-2.82)$ & 0.889 & $2.27(0.53-9.64)$ & 0.267 \\
\hline$>60$ & $55(20.8)$ & $26(17.4)$ & $29(25.2)$ & $0.67(0.24-1.85)$ & 0.443 & $1.41(0.25-7.84)$ & 0.696 \\
\hline \multicolumn{8}{|l|}{ Education } \\
\hline Low (high school) & $141(57.3)$ & $77(57.0)$ & $64(57.7)$ & 1.00 & & 1.00 & \\
\hline High (college/university) & $105(42.7)$ & $58(43.0)$ & $47(42.3)$ & $1.03(0.62-1.70)$ & 0.922 & $0.70(0.34-1.44)$ & 0.334 \\
\hline \multicolumn{8}{|l|}{ Occupation } \\
\hline No & $145(58.9)$ & $86(63.7)$ & $59(53.2)$ & 1.00 & & 1.00 & \\
\hline Yes & $101(41.1)$ & $49(36.3)$ & $52(46.8)$ & $0.65(0.39-1.08)$ & 0.095 & $0.63(0.32-1.22)$ & 0.167 \\
\hline \multicolumn{8}{|l|}{ Economic status } \\
\hline High & $14(6.1)$ & $7(5.5)$ & $7(6.8)$ & 1.00 & 0.585 & 1.00 & 0.278 \\
\hline Moderation & $177(76.6)$ & $96(75.0)$ & $81(78.6)$ & $0.71(0.35-1.44)$ & 0.343 & $0.49(0.20-1.19)$ & 0.117 \\
\hline Low & 40 (17.3) & $25(19.5)$ & $15(14.6)$ & $0.60(0.18-2.05)$ & 0.415 & $0.67(0.13-3.36)$ & 0.629 \\
\hline \multicolumn{8}{|l|}{ With partner } \\
\hline No & $23(8.7)$ & $14(9.4)$ & $9(7.8)$ & 1.00 & & 1.00 & \\
\hline Yes & $241(91.3)$ & $135(90.6)$ & $106(92.2)$ & $0.82(0.34-1.96)$ & 0.654 & $0.89(0.28-2.82)$ & 0.840 \\
\hline \multicolumn{8}{|l|}{ Children } \\
\hline No & $16(6.6)$ & $9(6.8)$ & $7(6.4)$ & 1.00 & & 1.00 & \\
\hline Yes & $226(93.4)$ & 124 (93.2) & $102(93.6)$ & $0.95(0.34-2.63)$ & 0.914 & $0.53(0.13-2.12)$ & 0.370 \\
\hline \multicolumn{8}{|l|}{ Smoking } \\
\hline No & $232(93.9)$ & $129(95.6)$ & $103(92.0)$ & 1.00 & & 1.00 & \\
\hline Yes & $15(6.1)$ & $6(4.4)$ & $9(8.0)$ & $0.53(0.18-1.54)$ & 0.246 & $0.60(0.15-2.37)$ & 0.462 \\
\hline \multicolumn{8}{|l|}{ Alcohol } \\
\hline No & $160(65.3)$ & $91(68.4)$ & 69 (61.6) & 1.00 & & 1.00 & \\
\hline Yes & $85(34.7)$ & $42(31.6)$ & $43(38.4)$ & $0.74(0.44-1.26)$ & 0.265 & $0.75(0.37-1.52)$ & 0.422 \\
\hline \multicolumn{8}{|l|}{ Postmenopause } \\
\hline No & $91(40.1)$ & $79(62.2)$ & $59(57.0)$ & 1.00 & & 1.00 & \\
\hline Yes & $136(59.9)$ & $48(37.8)$ & $43(43.0)$ & $0.81(0.47-1.37)$ & 0.427 & $0.92(0.32-2.63)$ & 0.872 \\
\hline \multicolumn{8}{|l|}{ Family history (breast cancer) } \\
\hline No & $209(83.9)$ & $112(82.4)$ & $97(85.8)$ & 1.00 & & 1.00 & \\
\hline Yes & $40(16.1)$ & $24(17.6)$ & $16(14.2)$ & $1.30(0.65-2.59)$ & 0.456 & $1.39(0.60-3.25)$ & 0.445 \\
\hline \multicolumn{8}{|c|}{ Family history (ovarian cancer) } \\
\hline No & $241(98.4)$ & $135(99.3)$ & $110(97.3)$ & 1.00 & & & \\
\hline Yes & $4(1.6)$ & $1(0.7)$ & $3(2.7)$ & $0.27(0.03-2.60)$ & 0.255 & NA & NA \\
\hline \multicolumn{8}{|l|}{ Family history (other cancers) } \\
\hline No & $137(56.4)$ & $71(53.4)$ & $66(60.0)$ & 1.00 & & 1.00 & \\
\hline Yes & $106(43.6)$ & $62(46.6)$ & $44(40.0)$ & $1.31(0.79-2.18)$ & 0.301 & $1.55(0.82-2.95)$ & 0.180 \\
\hline \multicolumn{8}{|l|}{ Clinical stage } \\
\hline Stage 0 & $61(23.1)$ & $40(26.8)$ & $21(18.3)$ & 1.00 & 0.133 & 1.00 & 0.504 \\
\hline Stage 1 & $123(46.6)$ & $62(41.6)$ & $61(53.0)$ & $0.53(0.28-1.01)$ & 0.053 & $0.62(0.23-1.66)$ & 0.340 \\
\hline Stage 2 & $80(30.3)$ & $47(31.5)$ & $33(28.7)$ & $0.75(0.38-1.49)$ & 0.409 & $0.56(0.21-1.50)$ & 0.248 \\
\hline Operating type & & & & & & & \\
\hline BCS & $178(67.4)$ & $93(62.4)$ & 85 (73.9) & 1.00 & & 1.00 & \\
\hline Mastectomy & $86(32.6)$ & $56(37.6)$ & $30(26.1)$ & $1.71(1.00-2.91)$ & 0.049 & $2.17(1.01-4.65)$ & 0.047 \\
\hline Axillary operating type & & & & & & & \\
\hline None & $17(6.4)$ & $11(7.4)$ & $6(5.2)$ & 1.00 & 0.585 & 1.00 & 0.710 \\
\hline SLNB & $243(92.0)$ & $135(90.6)$ & $108(93.9)$ & $0.68(0.24-1.90)$ & 0.465 & $0.62(0.23-1.66)$ & 0.408 \\
\hline ALND & $4(1.5)$ & $3(2.3)$ & $1(0.9)$ & $1.64(0.14-19.39)$ & 0.696 & NA & NA \\
\hline
\end{tabular}

$\mathrm{OR}=$ odds ratio; $\mathrm{Cl}=$ confidence interval; $\mathrm{NA}=$ not available; $\mathrm{BCS}=$ breast-conserving surgery; $\mathrm{SLNB}=$ sentinel lymph node biopsy; $\mathrm{ALND}=$ axillary lymph node dissection. 



Figure 2. Comparison of (A) distress thermometer and (B) Center for Epidemiological Studies-Depression scale (CES-D) average scores of three group; breast-conserving surgery $(B C S)(n=178)$, only mastectomy $(M)(n=57)$, and mastectomy with reconstruction $(M+R)(n=29)$. All three groups showed overall decreasing levels of distress as measured via the distress scales, and those in the reconstruction group showed the most reduction in distress at T3.
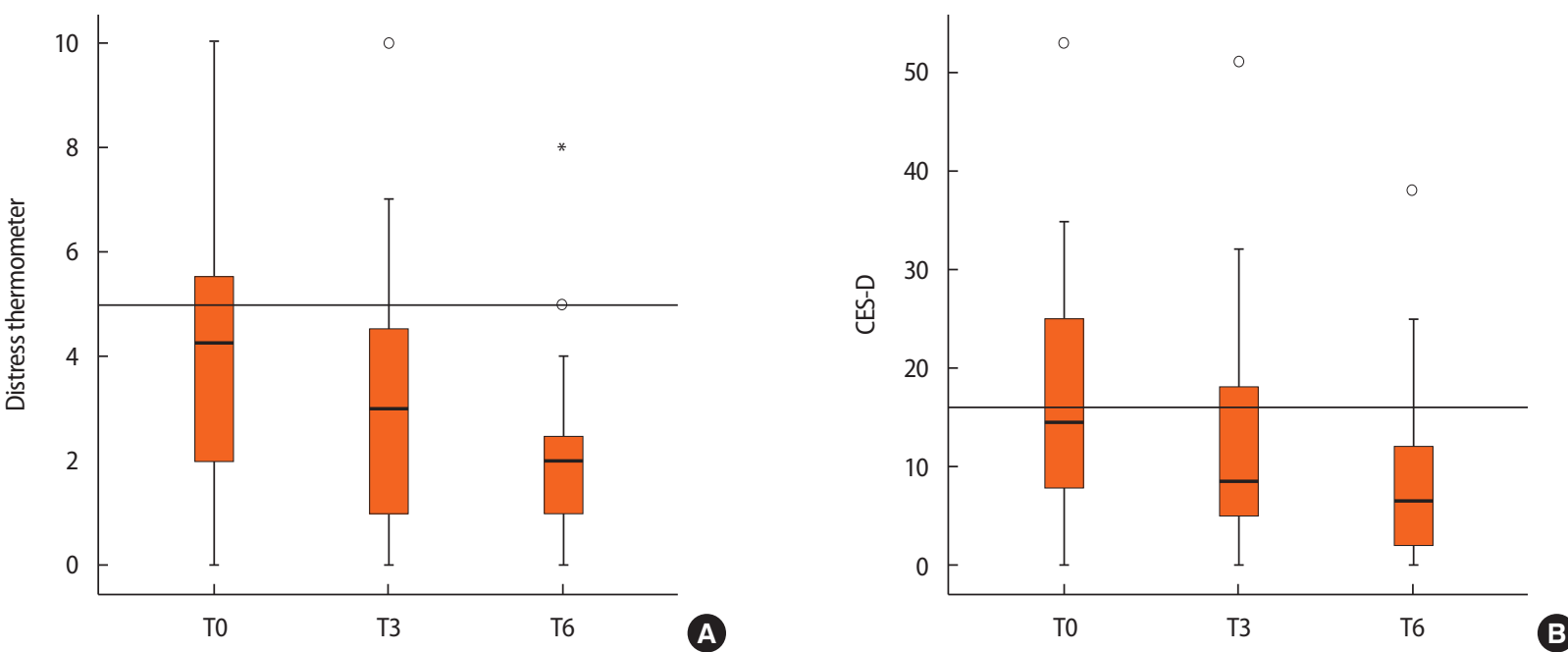

Figure 3. Difference in (A) distress thermometer (DT) and (B) Center for Epidemiological Studies-Depression scale (CES-D) scales according to the time flow in all patients $(n=264)$. Distress levels were highest immediately before surgery in breast cancer patients and gradually decreased as the treatment proceeded. The DT average score was 4.23 (SD, 2.71) at T0, 3.19 (SD, 2.56) at T3 ( $n=96)$, and 2.53 (SD, 2.13) at T6 ( $n=58)$. The CES-D average score was 17.55 (SD, 12.27) at T0, $12.85(\mathrm{SD}, 12.04)$ at T3, and 6.40 (SD, 8.24) at T6. o=outlier. * Extreme value.

Table 2. Changes in adjusted means of DT and CES-D scores in high distress group

\begin{tabular}{|c|c|c|c|c|c|c|c|}
\hline \multirow[b]{2}{*}{ Outcome } & \multicolumn{2}{|c|}{ TO $(n=149)$} & \multicolumn{2}{|c|}{ T3 $(n=53)$} & \multicolumn{2}{|c|}{$T 6(n=38)$} & \multirow[b]{2}{*}{$p$-value } \\
\hline & $\begin{array}{l}\text { Intervention } \\
\text { group } \\
(n=21)\end{array}$ & $\begin{array}{c}\text { Nonintervention } \\
\text { group } \\
(n=128)\end{array}$ & $\begin{array}{l}\text { Intervention } \\
\text { group } \\
(n=9)\end{array}$ & $\begin{array}{c}\text { Nonintervention } \\
\text { group } \\
(n=43)\end{array}$ & $\begin{array}{l}\text { Intervention } \\
\text { group } \\
(n=5)\end{array}$ & $\begin{array}{l}\text { Nonintervention } \\
\text { group } \\
(n=32)\end{array}$ & \\
\hline DT score & $7.30 \pm 1.76$ & $5.71 \pm 2.16$ & $5.78 \pm 3.08$ & $3.50 \pm 2.21$ & $3.80 \pm 2.71$ & $2.97 \pm 2.20$ & 0.051 \\
\hline CES-D score & $31.67 \pm 12.73$ & $24.00 \pm 9.55$ & $22.44 \pm 15.35$ & $15.26 \pm 12.00$ & $18.00 \pm 12.54$ & $9.97 \pm 7.92$ & 0.077 \\
\hline
\end{tabular}

Value are presented as mean \pm SD.

$\mathrm{DT}=$ distress thermometer; $\mathrm{CES}-\mathrm{D}=$ Center for Epidemiological Studies-Depression scale.

${ }^{*}$ Analysis also time points at T0, T3, and T6 by linear mixed models. 
great in breast cancer patients, as evidenced by more than half of the current study population experiencing it as such. Moreover, results indicated that the type of surgery that patients undergo is significantly associated with level of distress such that patients who were planning to undergo BCS had lower distress levels than those who were planning to undergo mastectomy. Initial distress levels declined rapidly when early reconstruction was performed in patients undergoing mastectomy. However, results showed an overall decrease in postoperative distress over time and did not find evidence for marked effects of the psychological intervention.

In this study, 56\% of patients experienced distress at the period of initial breast cancer diagnosis. The prevalence of distress was $41.0 \%$ (110/268) as measured by the DT and 48.1\% (129/268) via the CES-D. Although many different studies have defined various criteria regarding the cutoff score of the DT, a cutoff score of 5 was used for this study. When using the same cutoff score of 5 , the prevalence of distress was $34 \%$ in a study by Dabrowski et al. [14] and $41 \%$ in a study by Hegel et al. [4], which are both comparable prevalence rates to those observed in this study. The CES-D has been used for evaluating distress in six other studies, in which the average proportion of patients with distress was $22 \%$ (range, 13\%-59\%) [15], which was relatively low compared with our study. We used both the DT and CES-D for evaluating distress, as it is believed that using a combination of highly reliable tools results in better psychometric properties [16].

Factors related to distress in breast cancer patients are known to be very diverse. In the a review of completed by Zainal et al. [15], various factors were found to influence depression, including sociodemographic variables (e.g., lower income/poor financial status, lower education, young age, marital status, having more number of children at home), cancer-related factors (e.g., elapsed time since diagnosis, reduced chance of survival, comorbidity index), treatment-related factors (e.g., chemotherapy, mastectomy, using an alternative treatment), subject psychological factors, lifestyle factors, social support, and quality of life. Our study found that surgery type was related to distress such that mastectomy significantly increased distress levels compared with BCS [5,17]. Similarly, Rowland et al. [18] compared the emotional outcome of three groups depending on the operation type (BCS vs. mastectomy with reconstruction vs. mastectomy only) and found that patients who underwent BCS had the most positive outcomes in terms of body image, sexuality, and partnership compared to those who had mastectomy with reconstruction or mastectomy alone. In a study by Schain et al. [19], an immediate reconstruction reduced distress levels 35\% compared to delayed reconstruction after mastectomy. Mastectomy was the most important risk factor for distress, and immediate reconstruction reduced distress levels in those who underwent mastectomy.

In this study, we evaluated the effectiveness of a psychological intervention in high risk patients. Results indicated that average DT and CES-D scores were significantly higher in the intervention group compared to the non-intervention group, and high risk patients tended to choose intervention. Nonetheless, distress levels decreased in both groups as time passed. However, among the 169 high risk patients, only 53 were re-evaluated at T3 and 38 at T6. Given this, there is a possibility that patients with lower stress levels were more likely to participate in the survey, causing a selection bias. In addition, it was difficult to identify the effect of the psychological intervention due to only a small number of subjects having participated in it. However, other studies have found such interventions to have a positive effect. For example, Jassim et al. [20] analyzed the effects of a psychological intervention in nonmetastatic breast cancer patients and found that the intervention had effects on the following psychological outcomes: anxiety, depression, and mood disturbance. In addition, according to a study that conducted 11 years of follow-up, participating in a psychological intervention decreased the recurrence rate (hazards ratio [HR] of $0.55 ; p=0.034$ ), mortality from breast cancer (HR of 0.44 ; $p=0.016)$, and overall mortality (HR of $0.44 ; p=0.016)$ [21].

In general, it is well known that breast cancer patients have higher distress levels in the early stages after receiving the initial diagnosis [22-24]. For example, in a study by Andreu et al. [25], the distress level of 102 nonmetastatic breast cancer patients was evaluated by looking at four different time points: initial visit, surgery, time of definitive diagnosis, and time of administration of chemotherapy. The time of diagnosis showed the highest level of distress compared to later time points. Vos et al. [26] also found that, among early stage breast cancer patients, it was more effective to participate in a psychological intervention within 4 months of having surgery. Furthermore, our study showed that the prevalence of distress was higher in the early period, a period shortly after diagnosis, than 3 and 6 months after surgery. Therefore, these findings suggest that active management of distress should be implemented shortly after the initial diagnosis of breast 
cancer.

Our study has several limitations. We analyzed 264 patients at the initial screening phase, but only 96 and 57 were evaluated for distress levels at T3 and T6, respectively, due to the nature of voluntary participation. Most importantly, in the group receiving the intervention, only nine patients (T3) and five patients (T6) were evaluated. For this reason, it was difficult to determine the effect of intervention. If follow-up data on patients receiving this intervention were to be collected, it would be considered useful in determining the effectiveness of the psychological intervention in reducing the distress levels associated with breast cancer.

The present study only evaluated distress levels in early stage breast cancer patients since more advanced stages of cancer are well known to be associated with significantly high distress levels. We found that many patients suffered from psychological distress after receiving an initial cancer diagnosis, and distress seemed most severe in the early period, eventually decreasing across time. Therefore, it is important to identify high risk patients in order to manage their distress in the initial phase. In addition, continuous distress screening and management in high distress groups is necessary since these patients tend to have high level of distress, regardless of time. By setting a specialized psychological care program — via psychological management - in the course of breast cancer treatment, patients' quality of life will likely improve.

\section{CONFLICT OF INTEREST}

The authors declare that they have no competing interests.

\section{REFERENCES}

1. Korean Breast Cancer Society. Breast Cancer Facts \& Figures 2014. Seoul: Korean Breast Cancer Society; 2014.

2. Fallowfield L, Ratcliffe D, Jenkins V, Saul J. Psychiatric morbidity and its recognition by doctors in patients with cancer. Br J Cancer 2001;84:1011-5.

3. Zabora J, BrintzenhofeSzoc K, Curbow B, Hooker C, Piantadosi S. The prevalence of psychological distress by cancer site. Psychooncology 2001;10:19-28.

4. Hegel MT, Moore CP, Collins ED, Kearing S, Gillock KL, Riggs RL, et al. Distress, psychiatric syndromes, and impairment of function in women with newly diagnosed breast cancer. Cancer 2006;107: 2924-31.

5. Schubart JR, Emerich M, Farnan M, Stanley Smith J, Kauffman GL, Kass RB. Screening for psychological distress in surgical breast cancer patients. Ann Surg Oncol 2014;21:3348-53.

6. National Comprehensive Cancer Network. NCCN practice guidelines for the management of psychosocial distress. Oncology (Williston Park) 1999;13:113-47.

7. Trijsburg RW, van Knippenberg FC, Rijpma SE. Effects of psychological treatment on cancer patients: a critical review. Psychosom Med 1992;54:489-517.

8. Jacobsen PB, Jim HS. Psychosocial interventions for anxiety and depression in adult cancer patients: achievements and challenges. CA Cancer J Clin 2008;58:214-30.

9. Meyer TJ, Mark MM. Effects of psychosocial interventions with adult cancer patients: a meta-analysis of randomized experiments. Health Psychol 1995;14:101-8.

10. Holland JC, Jacobsen PB, Riba MB; NCCN Fever and Neutropenia Practice Guidelines Panel. NCCN: distress management. Cancer Control 2001;8(6 Suppl 2):88-93.

11. Barlow JH, Wright CC. Dimensions of the Center of Epidemiological Studies-Depression scale for people with arthritis from the UK. Psychol Rep 1998;83(3 Pt 1):915-9.

12. Hann D, Winter K, Jacobsen P. Measurement of depressive symptoms in cancer patients: evaluation of the Center for Epidemiological Studies Depression scale (CES-D). J Psychosom Res 1999;46: 437-43.

13. Beekman AT, Deeg DJ, Van Limbeek J, Braam AW, De Vries MZ, Van Tilburg W. Criterion validity of the Center for Epidemiologic Studies Depression scale (CES-D): results from a community-based sample of older subjects in the Netherlands. Psychol Med 1997;27: 231-5.

14. Dabrowski M, Boucher K, Ward JH, Lovell MM, Sandre A, Bloch J, et al. Clinical experience with the NCCN distress thermometer in breast cancer patients. J Natl Compr Canc Netw 2007;5:104-11.

15. Zainal NZ, Nik-Jaafar NR, Baharudin A, Sabki ZA, Ng CG. Prevalence of depression in breast cancer survivors: a systematic review of observational studies. Asian Pac J Cancer Prev 2013;14:2649-56.

16. Vodermaier A, Linden W, Siu C. Screening for emotional distress in 
cancer patients: a systematic review of assessment instruments. J Natl Cancer Inst 2009;101:1464-88.

17. Eversley R, Estrin D, Dibble S, Wardlaw L, Pedrosa M, FavilaPenney W. Post-treatment symptoms among ethnic minority breast cancer survivors. Oncol Nurs Forum 2005;32:250-6.

18. Rowland JH, Desmond KA, Meyerowitz BE, Belin TR, Wyatt GE, Ganz PA. Role of breast reconstructive surgery in physical and emotional outcomes among breast cancer survivors. J Natl Cancer Inst 2000;92:1422-9.

19. Schain WS, Wellisch DK, Pasnau RO, Landsverk J. The sooner the better: a study of psychological factors in women undergoing immediate versus delayed breast reconstruction. Am J Psychiatry 1985;142:40-6.

20. Jassim GA, Whitford DL, Hickey A, Carter B. Psychological interventions for women with non-metastatic breast cancer. Cochrane Database Syst Rev 2015;(5):CD008729.

21. Andersen BL, Yang HC, Farrar WB, Golden-Kreutz DM, Emery CF, Thornton LM, et al. Psychologic intervention improves survival for breast cancer patients: a randomized clinical trial. Cancer 2008;
$113: 3450-8$.

22. Fiszer C, Dolbeault S, Sultan S, Brédart A. Prevalence, intensity, and predictors of the supportive care needs of women diagnosed with breast cancer: a systematic review. Psychooncology 2014;23:361-74.

23. Khan F, Amatya B, Pallant JF, Rajapaksa I. Factors associated with long-term functional outcomes and psychological sequelae in women after breast cancer. Breast 2012;21:314-20.

24. Akechi T, Okuyama T, Endo C, Sagawa R, Uchida M, Nakaguchi T, et al. Patient's perceived need and psychological distress and/or quality of life in ambulatory breast cancer patients in Japan. Psychooncology 2011;20:497-505.

25. Andreu Y, Galdón MJ, Durá E, Martínez P, Pérez S, Murgui S. A longitudinal study of psychosocial distress in breast cancer: prevalence and risk factors. Psychol Health 2012;27:72-87.

26. Vos PJ, Visser AP, Garssen B, Duivenvoorden HJ, de Haes HC. Effects of delayed psychosocial interventions versus early psychosocial interventions for women with early stage breast cancer. Patient Educ Couns 2006;60:212-9. 